\title{
Application of SNP array for rapid prenatal diagnosis: implementation, genetic counselling and diagnostic flow
}

\author{
Malgorzata Srebniak $^{\star, 1}$, Marjan Boter ${ }^{1}$, Grétel Oudesluijs ${ }^{1}$, Marieke Joosten ${ }^{1}$, Lutgarde Govaerts ${ }^{1}$, \\ Diane Van Opstal ${ }^{1}$ and Robert-Jan H Galjaard ${ }^{1}$
}

We report on the validation and implementation of the HumanCytoSNP-12 array (Illumina) (HCS) in prenatal diagnosis. In total, 64 samples were used to validate the Illumina platform (20 with a known (sub) microscopic chromosome abnormality, 5 with known maternal cell contamination (MCC) and 39 normal control samples). There were no false-positive or false-negative results. In addition to the diagnostic possibilities of arrayCGH, the HCS allows detection of regions of homozygosity (ROH), triploidy and helps recognising MCC. Moreover, in two cases of MCC, a deletion was correctly detected. Furthermore we found out that only about $\mathbf{5 0} \mathrm{ng}$ of DNA is required, which allows a reporting time of only 3 days. We also present a prospective pilot study of 61 fetuses with ultrasound abnormalities and a normal karyotype tested with HCS. In 4 out of 61 (6.5\%) fetuses, a clinically relevant abnormality was detected. We designed and present pre-test genetic counselling information on categories of possible test outcomes. On the basis of this information, about $90 \%$ of the parents chose to be informed about adverse health outcomes of their future child at infancy and childhood, and $55 \%$ also about outcomes at an adult stage. The latter issue regarding the right of the future child itself to decide whether or not to know this information needs to be addressed. European Journal of Human Genetics (2011) 19, 1230-1237; doi:10.1038/ejhg.2011.119; published online 22 June 2011

Keywords: genomic SNP array; rapid genomic array testing; whole-genome screening; pre-test genetic counselling; prenatal diagnosis

\section{INTRODUCTION}

Array-based comparative genomic hybridisation (arrayCGH) has already been successfully used in prenatal diagnosis. ${ }^{1-7}$ Although arrayCGH cannot detect regions of homozygosity $(\mathrm{ROH})$ and has a limited value for detecting triploidy and maternal cell contamination (MCC), single-nucleotide polymorphism (SNP) arrays are still not routinely used for prenatal diagnosis in ongoing pregnancies. There are only two studies using a whole-genome SNP array on fetal material. Tyreman et $a l^{8}$ have performed a retrospective analysis on 106 karyotypically normal referrals with abnormal ultrasound findings using the GeneChip 6.0 array from Affymetrix (Santa Clara, CA, USA). Faas et al ${ }^{9}$ have also shown results of a SNP array (Affymetrix $250 \mathrm{k} \mathrm{NspI}$ ) on prenatal material. In both studies all analyses were performed after 24th week of pregnancy, termination of pregnancy (TOP), intrauterine fetal death or birth on DNA isolated from fetal or neonatal material, hence, rapid analysis was not required.

Prenatal genetic diagnosis after ultrasound detection of fetal abnormalities prefers a fast diagnostic technique, especially when there is a limited legal time frame for TOP. The standard cytogenetic prenatal diagnosis includes rapid detection of specific abnormalities (most common aneuploidies and known microdeletions) and timeconsuming karyotyping. Decisions regarding pregnancy management are best made taking into account both ultrasonographic and genetic results. Implementing rapid microarray technology in routine prenatal diagnosis will allow the detection of unbalanced chromosomal abnormalities in a shorter time frame with much better resolution than conventional karyotyping.

We report on the validation and implementation of a genomic SNP array (HumanCytoSNP-12, Illumina - HCS) (San Diego, CA, USA) in prenatal diagnosis. To our best knowledge it is the first report describing the implementation of rapid whole-genome SNP array analysis in ongoing pregnancies.

\section{MATERIALS AND METHODS}

Pilot study design

From May 2009 to November 2010, the HCS microarray from Illumina was performed on 125 prenatal samples. The 12-sample HCS BeadChip is a whole-genome scanning panel. It includes 300 000 markers genome-wide tag SNPs and markers targeting all regions of known cytogenetic importance. This includes dense coverage of around 250 genomic regions commonly screened in cytogenetic laboratories, including subtelomeric regions, pericentromeric regions, sex chromosomes and targeted coverage in around 400 additional disease-related genes (http://www.illumina.com).

Validation was carried out on 64 prenatal samples, which all were karyotyped before (Table 1). Normal controls were patients referred because of advanced maternal age or abnormal first-trimester screening. In these cases, DNA was isolated from $\sim 4 \mathrm{ml}$ amniotic fluid (AF) and also used for rapid aneuploidy detection (RAD). DNA remaining after routine diagnostics was used to perform the array assay. That is the reason why some samples had very low DNA input. Samples with low DNA input were not excluded from validation to test whether HCS can be performed on patients with 
Table 1 The HCS validation results

\begin{tabular}{|c|c|c|c|}
\hline Group & $\mathrm{N}$ & Chromosome constitution & HCS results \\
\hline Known abnormal samples & 25 & & \\
\hline $\begin{array}{l}\text { Known autosomal chromosome } \\
\text { aberration cases (targeted } \\
\text { analysis) }\end{array}$ & $\begin{array}{l}13 \text { (UAF, } \\
\text { CAF or LTC) }\end{array}$ & $\begin{array}{l}\text { T13; T18; T21; del(13)(q22q31); } \\
\text { del(18)(p11.32p11.23); del(9)(q34); } \\
\text { del(22)(q11q11); der(18)t(16;18)(p13.3;p11); } \\
\text { del(7)(q3?5); del(13)(q21.1q21.3); } \\
\text { inv(13)(q13q21.3), 69,XXX; 69,XXY. }\end{array}$ & All confirmed, except for the balanced inversion (12/13) \\
\hline $\begin{array}{l}\text { Blood-stained samples with } \\
\text { suspected MCC }\end{array}$ & 5 (UAF) & $\begin{array}{l}\text { 17q24 2,7Mb loss; del(13)(q22q31); } \\
1 \times 46, X X, 2 x 46, X Y\end{array}$ & $\begin{array}{l}\text { MCC correctly identified (5/5), both deletions called by } \\
\text { Nexus } 5.0\end{array}$ \\
\hline Normal control samples & 39 & & \\
\hline $\begin{array}{l}\text { Normal control patients (AMA } \\
\text { and/or FTS) (whole genome } \\
\text { analysis) }\end{array}$ & 39 (UAF) & Normal karyotypes & 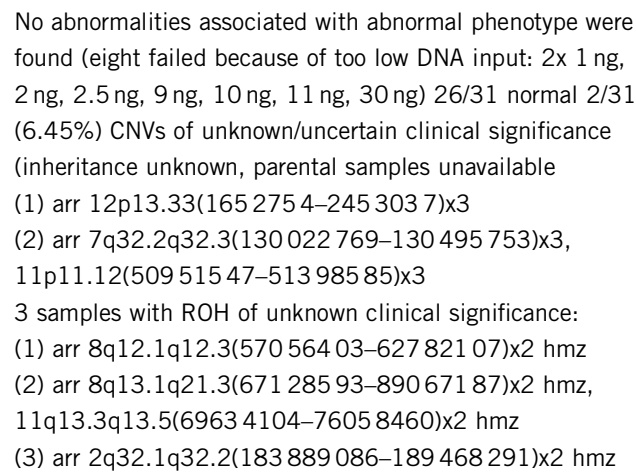 \\
\hline
\end{tabular}

Abbreviations: AMA, advanced maternal age; CAF, cultured amniotic fluid; FTS, abnormal first-trimester screening; LTC, long-term culture chorion villi; MCC, maternal cell contamination; $\mathrm{POL}$, polymorphism; ROH, region of homozygosity; T, trisomy; $u A F$, uncultured amniotic fluid; UV, unknown variants.

NB: Physical positions of the breakpoints utilise the hg18 build of the human genome sequence.

very low DNA concentrations. In the control cases, parental DNA was unavailable.

In order to determine whether HCS can detect MCC, five blood-stained uncultured AF ( $\mathrm{uAF}$ ) samples were tested (suspected MCC, including a sample fraction of one index patient not used for array testing). Suspected MCC was not routinely measured as those samples were cultured and not used for RAD.

In total, 61 index fetuses with ultrasound abnormalities (Table in Supplementary Material) that were referred for array testing were examined with HCS. Overall, 14 patients were referred after TOP or after birth (retrospective testing), and 47 fetuses were tested prospectively in an ongoing pregnancy. Some of them terminated the pregnancy before receiving the array results. The mean gestational age of the fetuses was 19 weeks (range 11-30 weeks). In all cases of ultrasound abnormalities detected in the second trimester, $30 \mathrm{ml} \mathrm{AF}$ was sampled for prenatal testing $(20 \mathrm{ml}$ was used for culturing and $10 \mathrm{ml}$ was used for DNA isolation).

Only in three cases parental DNA was not available: case 4 - the parents refused to be tested; case 5 - a pathogenic deletion unlikely inherited from a parent was found; case 11 - the father refused to be tested.

In the index group, only clear AF samples were used for array testing, green/ brown or blood-stained samples were excluded because of possible MCC.

\section{Sample preparation}

A volume of 4-10 $\mathrm{ml}$ of $\mathrm{uAF}$ was used for DNA isolation with the Chemagic Magnetic Separation Module I (Chemagen, BaesWeiler, Germany). The DNA yield from uAF ranged between $10 \mathrm{ng}$ and $500 \mathrm{ng}$. DNA from uAF was first used for RAD.

For DNA isolation of cultured cells, the midi kit from Qiagen (Hilden, Germany) was used, resulting in about $7-26 \mu \mathrm{g}$ DNA per sample.

DNA concentration was measured by using a Quant-it DNA assay kit (broad range, 2-1000 ng) (Invitrogen, Paisley, UK). Depending on the DNA concentration, $4-10 \mu \mathrm{l}$ volume of patient DNA was used for the array assay.

\section{SNP array, data analysis and interpretation}

During validation, $1-200 \mathrm{ng}$ of DNA was used as an input for a single array. DNA amplification, tagging and hybridisation were performed according to the manufacturer's protocol. The array slides were scanned on an iScan Reader (Illumina). Data analysis was performed using GenomeStudio version 2010.1, KaryoStudio version 1.2 (Illumina, standard settings) and Nexus Copy Number 5.0 (BioDiscovery, El Segundo, CA, USA). The HapMap control set provided by the manufacturer was used as a control. After validation, 50-200 ng of DNA was used (if possible) and the samples were only analysed by using Nexus.

Standard settings for SNP arrays in Nexus were adjusted: a cutoff value of 0.15 , homozygous frequency threshold of 0.95 and minimum loss of heterozygosity (region with LOH) length of $5000(\mathrm{~kb})$ were set (SNP-FASST Rank Segmentation). QC measurement in Nexus was used as a measure of the array profile quality. Samples with $\mathrm{QC}<0.13$ were further analysed.

The B allele frequency (BAF) of HCS helps to recognise and exclude falsepositive calls. The BAF is a value between 0 and 1 , and represents the proportion contributed by one SNP allele (B) to the total copy number. A BAF value of 0.5 indicates a heterozygous genotype $(\mathrm{AB})$, whereas 0 and 1 indicate homozygous genotypes (AA and $\mathrm{BB}$, respectively). For example, a region with a deletion in all cells will show homozygosity - bands at 1 and 0 . In other words, a loss should always be accompanied by a LOH visible in the BAF plot. A region of single-copy-number gain in all cells will, in addition to the two bands of homozygous SNPs at $\mathrm{BAF}=0$ (AAA) and $\mathrm{BAF}=1(\mathrm{BBB})$, also show two bands: one at $\mathrm{BAF}=0.33$ with $\mathrm{SNPs}$ having genotype $\mathrm{AAB}$ and one at $\mathrm{BAF}=0.67$ with SNPs having genotype ABB.

The following resolution was generally applied: losses $\geq 150 \mathrm{~kb}$ and gains $\geq 200 \mathrm{~kb}$.

UCSC built Hg18 (Human Mar. 2006 (NCBI36/hg18) Assembly) was used to analyse the data. Copy number variations (CNVs) were checked in the local databases, DGV (http://projects.tcag.ca/variation/), CHOP database (http:// cnv.chop.edu) and literature. 
Parental DNA, when available, was analysed to identify inherited CNVs. Rare CNVs not associated with an increased risk or presence of abnormal phenotype, inherited from a healthy parent, were assumed to be benign. If a similar gain or loss was found in $\geq 3$ healthy individuals (preferably in different studies), the call was recognised as a polymorphism (POL). The most important criterion for classification as mutagenic (MUT) is association with a known abnormal phenotype. There was no cutoff for the size of the array abnormality. CNVs associated with an abnormal phenotype were assumed to be MUT. Also CNVs associated with an abnormal phenotype of variable penetrance or with a known increased risk of an abnormal phenotype were classified as MUT. ROHs are almost always of unknown clinical relevance, except the cases in which they indicate (segmental or whole chromosome) uniparental disomy of imprinted regions. A de novo (or of unknown inheritance) gain and loss (CNV) or copy neutral $\mathrm{ROH}$ of unknown (absence of a known disease-causing gene) or uncertain clinical importance were defined as unclassified variants (UVs).

All MUTs were reported to the referring clinical geneticist. UVs were not reported, but in some cases (uncertain relevance) were discussed with the referring clinical geneticist. Common POL and inherited (benign) rare CNVs were not reported. In this study, only clinically relevant CNVs were confirmed with another technique in order to validate HCS calls. We also followed recommendations of The Association for Clinical Cytogenetics (Constitutional Array CGH Best Practice Guidelines, http://www.cytogenetics.org.uk/prof_ standards/professional_standards.htm).

After validation we decided to use an alternative technique to determine whether a specific imbalance is a true-positive call only in cases in which the possibility existed that an imbalance is not a true CNV. Clear imbalances do not require confirmatory studies. ${ }^{10}$

Parental arrays were only used to determine the origins of fetal CNVs. Parental whole-genome analysis was, therefore, not performed to avoid incidental findings.

\section{Pre-test genetic counselling}

The gynaecologist referred pregnant women and their partner (=parents) with abnormal fetal ultrasound findings to the clinical geneticist. SNP array analysis was offered when a submicroscopic chromosomal aberration was included in the differential diagnosis. We designed a form in which categories of possible outcomes of the fetal microarray were described (Table 2). Pre-test genetic counselling included general information about cytogenetics (RAD and conventional karyotyping), molecular genetics, a tailored description of the array test, benefits and limitations of the offered tests and finally discussion of all possible test outcomes. The potential detection of variations of unknown or uncertain clinical relevance (UVs) was mentioned as well. Parents were told that these results would not be revealed to them. After genetic counselling they decided which test results they wish to receive (Table 2). During pre-test genetic counselling, we discuss the very small chance of a genetic finding affecting the health of the parents/family members. For this validation study, we did not include this as a separate category in the informed consent form, as parental arrays were only used in a targeted way to determine the origin of fetal CNVs. In future editions, we will include this choice to have a more complete record. The parents could decide whether they wished to be contacted if clinical significant information about their child would become available in the future. A signed informed consent with the parents' choices was archived. Parental blood for determination of de novo versus inherited variations was preferably sampled on the day of counselling or at the time of prenatal sampling.

\section{RESULTS}

Figure 1 shows our current diagnostic flow. Now we choose to perform genomic array testing directly after RAD on uAF samples. Parental and fetal DNA are simultaneously analysed. The whole-array assay (from DNA isolation to an array result) can be performed within $72 \mathrm{~h}$ (3 days), if required. In general, it takes 1 week mainly depending on the day of sampling.

During validation 18 out of 20 known chromosome abnormalities were detected using HCS. The results of the validation samples are
Table 2 Pre-test genetic counselling: possible outcomes of the genomic array examination and choices of the parents (after pre-test counselling and before array analysis)

Possible outcomes and the meaning of an abnormality seen in array analysis

1. No evidence of known disease-causing abnormality

2. Abnormal result that explains the ultrasound abnormality

3. Abnormal result that does not explain the ultrasound abnormality, however, an adverse effect on the growth and/or development of the child is (most probably) expected. Sometimes the nature or severity of the defects/disease cannot be predicted

4. Abnormal array results that (most probably) have an adverse health effect in adulthood

5. Inherited DNA variation of yet unknown significance ( $\sim 10 \%$ of results): little cause for concern unless the parent has a congenital anomaly/developmental disorder

6. New DNA variation (not inherited) of unknown significance ( $\sim 10 \%$ of results). Sometimes its meaning becomes clear in the future

\section{Choices of the parents}

More than one option is possible

$\%$ Of parents who have chosen the particular option

1. Only an abnormal result that explains $11.1 \%$ ultrasound abnormality

2. Results that probably have an adverse health effect in infancy and childhood

3. Results that probably have an adverse health effect in adulthood

4. I would like to be informed later when clinically important information becomes available

5. Results that probably have an adverse effect on my own health

$88.9 \%$

$55.5 \%$

$61.1 \%$

Discussed during pre-test counselling together with category 3 , but not yet included as a separate category in the informed consent form.

summarised in Table 1. As expected, the samples with a balanced inversion and AZFc microdeletion showed normal results. Triploidy was correctly identified (Figure 2 upper panel). All blood-stained samples with suspected MCC were identified by HCS (compare a normal profile on Figure 2 middle panel and a profile with MCC on Figure 2 lower panel). Moreover, we tested two samples in which a deletion and MCC were present (validation sample with a 10.2-Mb $\operatorname{del}(13)(\mathrm{q} 22 \mathrm{q} 31)$, and a blood-stained fraction of a patient's sample with a 2.7-Mb del(17)(q24.3q24.3)). Despite MCC, which was easy to recognise in the BAF plot in both samples, the deletions were correctly detected by Nexus 5.0 (see Figure 3 for $17 \mathrm{q}$ deletion). As both deletions were not called by GenomeStudio and KaryoStudio, Nexus 5.0 was chosen as a primary analysis tool after we have validated it in a prenatal setting.

According to the manufacturer, the HCS requires input of $200 \mathrm{ng}$ DNA. However, we have seen that samples with a very low DNA input can also show a good quality array profile (see Supplementary Material). In general, we estimate that samples with DNA input $<50 \mathrm{ng}$ are at risk of failure, but we do not exclude them from array analysis.

Eight normal control samples showed a low-quality $(\mathrm{QC}>0.13)$ array profile probably because of low DNA input $(<30 \mathrm{ng})$. There were no pathogenic abnormalities found in the normal control group. In 5 out of 31 normal control samples, UVs were detected. Two $(6.45 \%)$ of them were CNVs en $3 \mathrm{ROH}>5 \mathrm{Mb}$ (see Table 1 ). 


\section{Diagnostic flow}

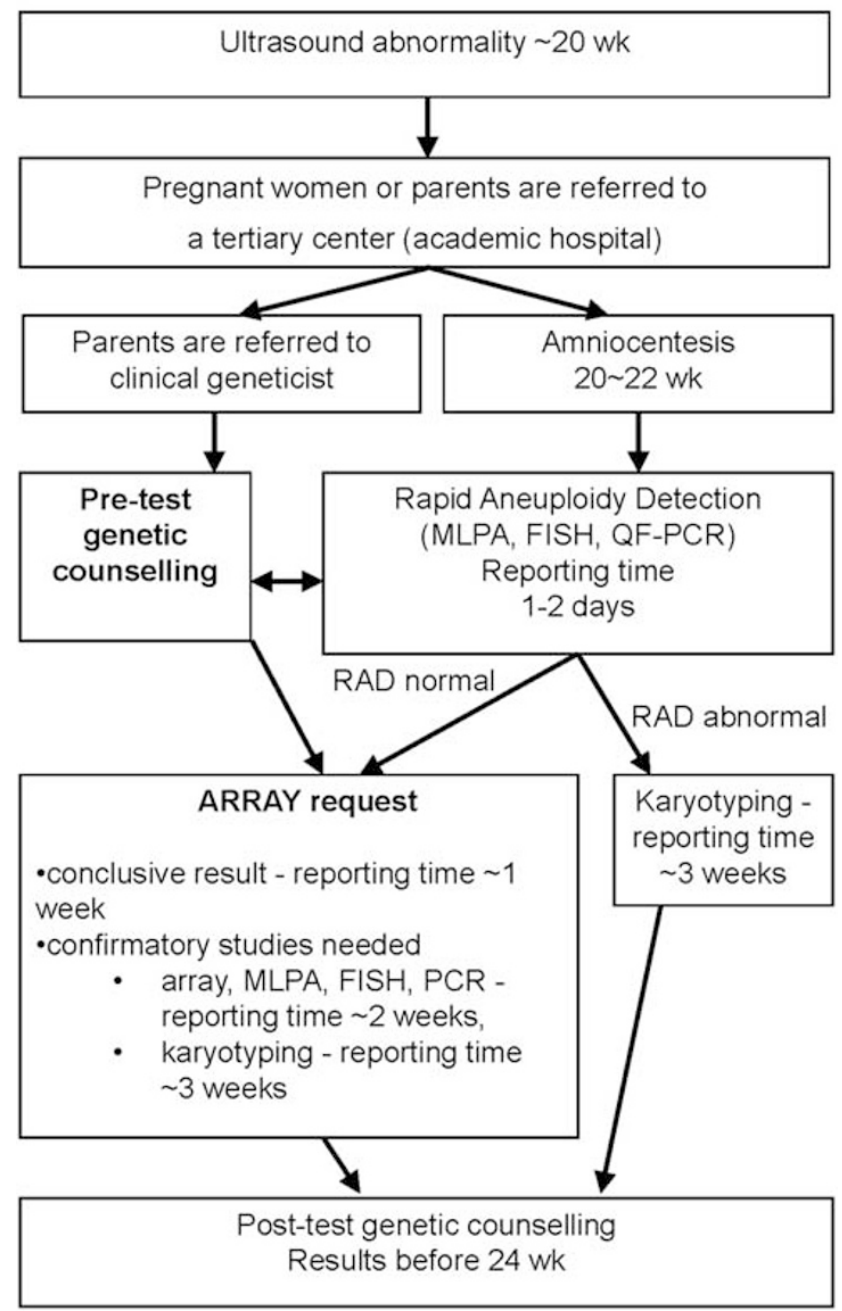

Figure 1 Current diagnostic flow.

The inheritance of the CNVs is unknown, as parental samples were not available for these normal validation samples.

Overall, 61 index patients were tested with HCS. The results of index patients are summarised in Supplementary Material. In all cases, a normal karyotype was found during routine chromosome analysis. Most of the samples were karyotyped before the array was requested. Since September 2010 we have performed a genomic SNP array immediately after sampling. Only one index patient (case 2) showed no results probably because of low DNA input (40 ng).

Out of 61 there were $4(6.5 \%)$ pathogenic abnormalities found:

\section{Case 4}

A 930-kb deletion in band 15q11.2, associated with an increased risk for seizures and behavioural disturbances, was detected. ${ }^{11-13}$ The deletion was confirmed with MLPA kits 181 and 182 (MRC-Holland, Amsterdam, The Netherlands) (data not shown). Parental DNA was unavailable. This finding belongs to category 3-abnormal result that does not explain the ultrasound abnormality, however, an adverse effect of the CNV on seizures and behavioural disturbances could be expected.
In this case, the array was requested after karyotyping was completed and before receiving the array results, the parents decided to terminate of the pregnancy based on the ultrasound findings (NT $5.5 \mathrm{~mm}$, atrio-ventricular defect (ASVD), microcephaly). The parents were counselled that this finding did not explain this combination of fetal ultrasound anomalies, but it is a risk factor for seizures and behavioural disturbances. The father and paternal uncle were known with seizures. A further investigation in the parents was not performed on their request. Presumably this $15 \mathrm{q} 11.2$ deletion is paternal.

\section{Case 5}

A $2.7-\mathrm{Mb}$ deletion in band $17 \mathrm{q} 24.3$ associated with campomelic dysplasia was found. ${ }^{14}$ The deletion was confirmed with Agilent $105 \mathrm{~K}$ array (data not shown). Parental DNA was not tested because a pathogenic deletion unlikely inherited from a parent was found. This finding belongs to category 2-abnormal result that explains the ultrasound abnormality (femoral bowing, possible skeletal dysplasia).

\section{Case 51}

An atypical $591 \mathrm{~kb}$ deletion (LCRC-LCRD) in band 22q11 associated with DiGeorge syndrome was found. The deletion was confirmed by MLPA kit P250 (data not shown). Exactly the same deletion was found in the maternal DNA sample. A patient with CATCH22 syndrome and a similar deletion has been published by Kurahashi et al. ${ }^{15}$ This finding belongs to category 3-abnormal result that does not explain the ultrasound abnormality, however, an adverse effect can be expected.

In this case, the array was requested after karyotyping and before receiving the arrays results, the parents decided to terminate of the pregnancy based on the clinical severity of the ultrasound findings (plexus choroïdeus cyst, increased NT, echodens focus heart, unilateral pes equinovarus, intrauterine growth retardation (IUGR), oligohydramnion). The parents were counselled that this finding did not explain all of the ultrasound anomalies. This finding is associated with DiGeorge syndrome; therefore, the mother will be investigated for features of DiGeorge syndrome. Further investigations to the aetiology of the ultrasound abnormalities are still pending.

\section{Case 60}

A $2.1-\mathrm{Mb}$ de novo deletion (overlapping with the commonly deleted region LCRA-LCRB-LCRC) in band 22q11 associated with DiGeorge syndrome was found in a fetus with single umbilical artery, extreme IUGR, echodens bowel and pericardial effusion. The deletion was confirmed by MLPA kit P250 (data not shown). This finding belongs to category 3-abnormal result that does not explain this combination of ultrasound anomalies, however, an adverse effect can be expected.

HSC showed 4-15 ROHs $>5 \mathrm{Mb}$ in three fetuses of consanguineous parents (cases 10, 52 and 61). In three other cases, a $\mathrm{ROH}$ was detected (cases 45, 55 and 58), but the parents were not consanguineous.

In 6 out of $61(9.8 \%)$ samples, a CNV of unknown clinical importance (UV) was detected (see Supplementary Material cases 6, $11,13,50,55,61)$.

Owing to the complexity concerning possible outcomes of microarrays in a prenatal diagnostic setting, we designed a consent form in which they are categorised and discussed with the parents during pre-test genetic counselling (Table 2). Currently, owing to this complexity, we only accept referrals from clinical geneticists for prenatal microarray analysis. Evaluation of the parents' choices showed that most of them were interested in results that (most probably) have an adverse health effect in infancy and childhood ( $\sim 90 \%)$. About half of the couples $(\sim 55 \%)$ chose to be informed 

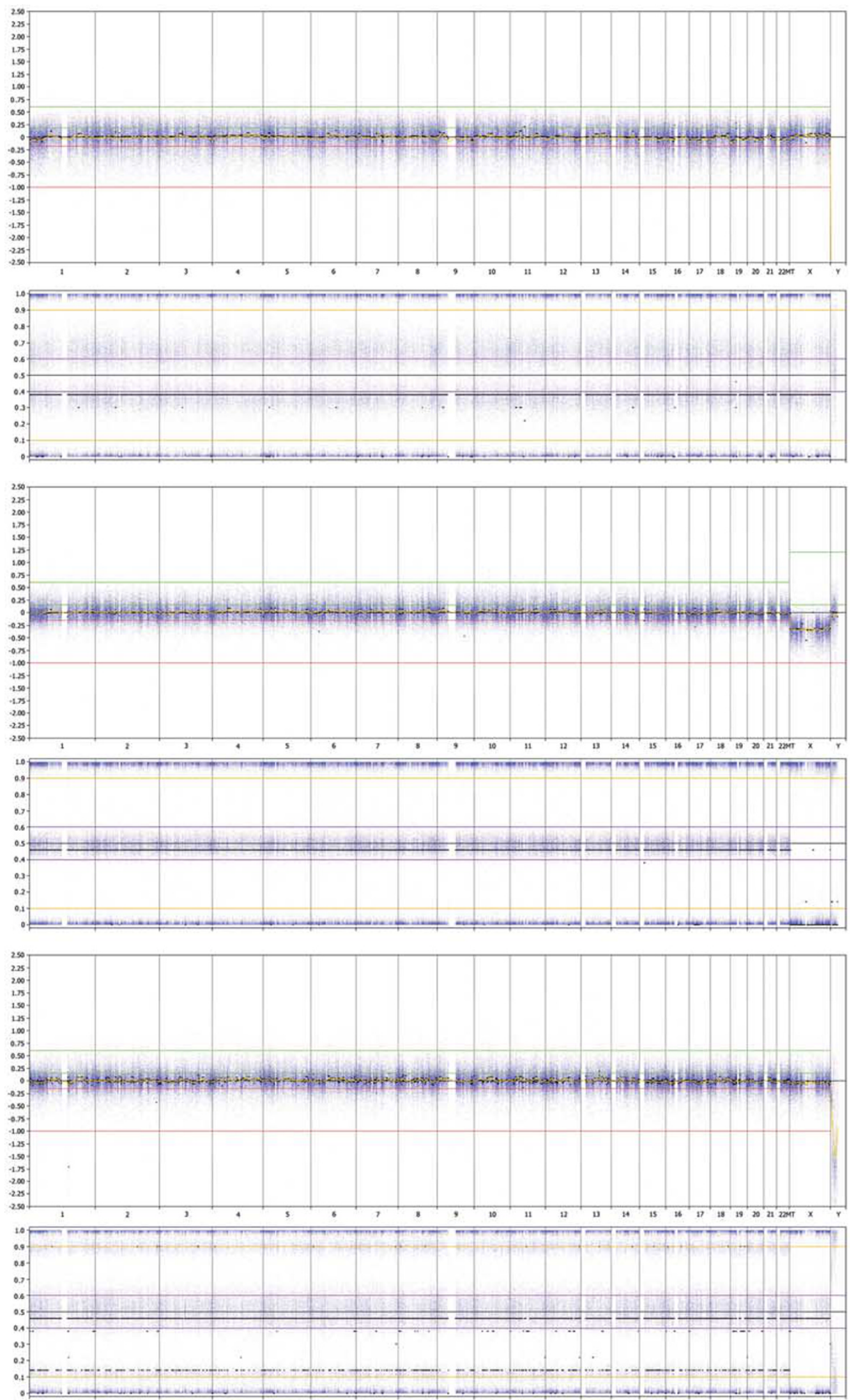

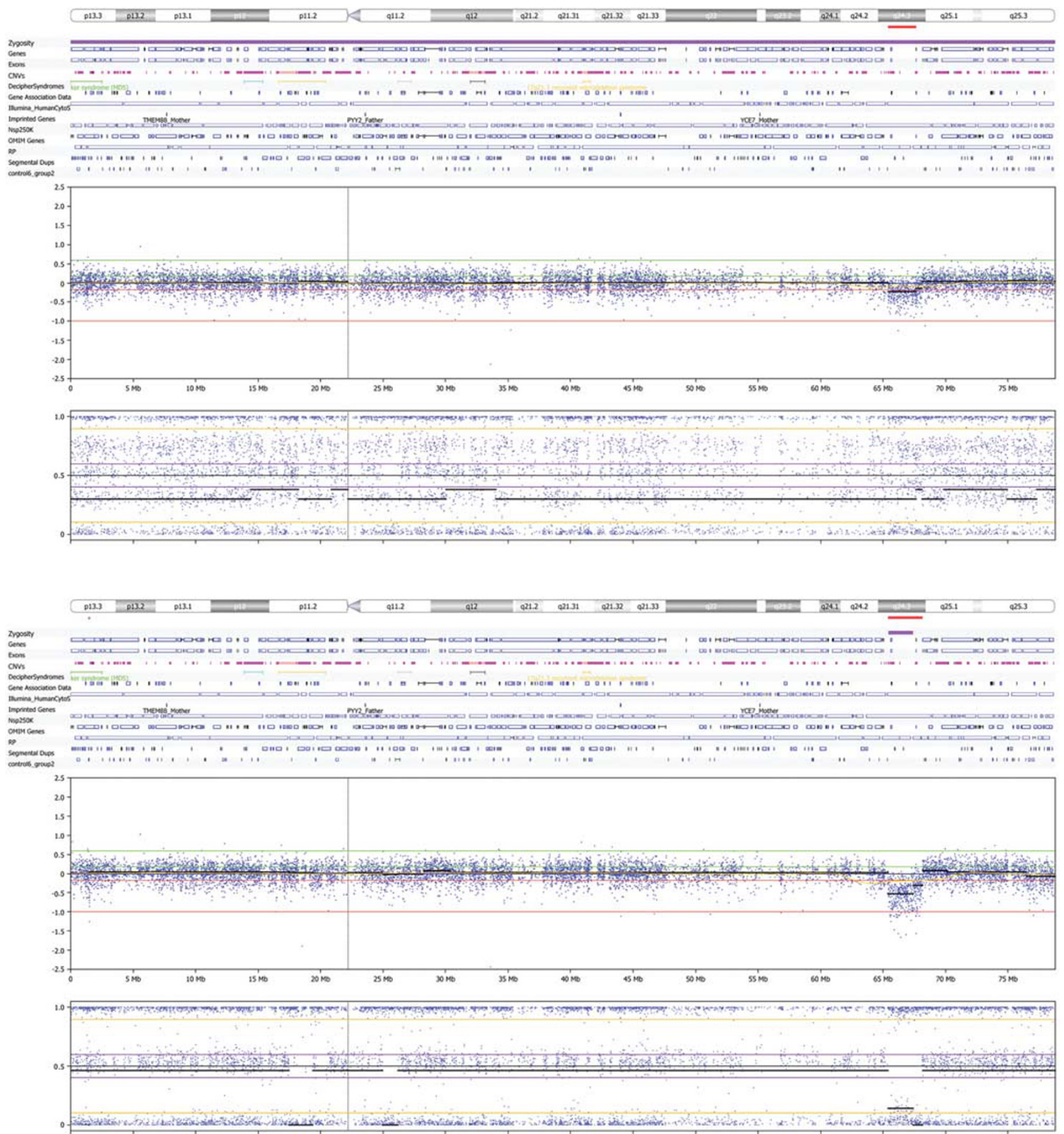

Figure 3 The $17 q$ deletion in a sample with MCC (case 5, Supplementary Material) shown on a chromosome plot from Nexus 5.0. The deletion in 17q24.3 was correctly identified by Nexus 5.0 in both clear and blood-stained AF. The upper panel shows the chromosome 17 plot of a sample with a $17 \mathrm{q}$ deletion and with MCC (uncultured blood-stained AF, DNA input $118 \mathrm{ng}, \mathrm{QC}=0.067$ ). The lower panel shows the chromosome 17 plot of the same patient sample with a $17 \mathrm{q}$ deletion, but the array assay is performed on uncultured clear AF (DNA input $54 \mathrm{ng}, \mathrm{QC}=0.07$ ).

Figure 2 Whole genome view of Human CytoSNP-12 profiles in Nexus 5.0. Both log ratios (above) and B allele frequencies (BAF) (below) is shown. The upper panel shows a profile of triploidy 69,XXX. The log ratio is normal; however, the BAF indicates a duplication of all chromosomes. There is no normal frequency at $\sim 0.5$, but two at $\sim 0.4$ and $\sim 0.6$. The DNA input was $117 \mathrm{ng}$, the $Q C=0.071$. The middle panel shows a normal male profile. The log ratio is about 0 (except for $\mathrm{X}$ ) and the BAF shows three lines (upper and lower ( 1.0 and $\sim 0.0)$ - homozygosity, middle ( 0.5) - heterozygosity). The DNA input was $164 \mathrm{ng}$, the $\mathrm{QC}=0.038$. The lower panel presents a profile with an indication for MCC. BAF shows additional BAF at $\sim 0.1$ and $\sim 0.9$ on all chromosomes. The DNA input is $202 \mathrm{ng}$, the $\mathrm{QC}=0.047$. 
about findings that affect the future health of their unborn child at an adult stage as well (Table 2). We considered the a priori chance of finding a genetic imbalance with clinical consequences for the parent(s)/family member(s) to be very small as parental arrays are only screened for fetal CNVs in a targeted way. In the present study we did not find any CNV of this kind.

\section{DISCUSSION}

In the Netherlands, a second trimester fetal ultrasound examination is offered to all pregnant women around the 20th week of gestation, generally performed by a midwife. Parents are referred to a gynaecologist if abnormalities are detected. Subsequent amniocentesis is mostly performed at about 20-22 weeks of gestation. The legal time frame for TOP in the Netherlands is before the 24th week of gestation. Before the implementation of HCS we used the $105 \mathrm{~K} / 180 \mathrm{~K}$ oligo-array from Agilent for whole-genome analysis in prenatal diagnosis (validation on 20 samples and after implementation 85 index patients tested - data not published). Owing to the need for AF cell culturing, it was often not feasible to produce a result before the 24th week of gestation. At that time (2007-2009), we did not choose to perform pre-test whole-genome amplification of DNA from uAF cells because of the chance of selective amplification bias. ${ }^{16}$ We noticed that in many cases, genomic array results did not contribute to the parents' decision about termination or continuation of the pregnancy because the reporting time was too long, or the array was requested after karyotyping appeared to be normal (eg, cases 4 and 51). As parents confronted with an abnormal result need time to consider whether or not to continue their pregnancy, only a 1- to 2-week-reporting time of genetic testing is acceptable, and the array should be requested immediately after amniocentesis. As HCS (Illumina) requires only 50 ng DNA, implementing HCS in prenatal diagnostics allowed using $\mathrm{uAF}$ cells for direct array analysis, which shortened the reporting time to 1 week or even 3 days if required.

In our experience, HCS allows detecting unbalanced chromosome abnormalities with a resolution of $150-200 \mathrm{~kb}$. We demonstrated that the HCS detected all-known unbalanced abnormalities (inclusive triploidy), except for an AZFc microdeletion. Samples with MCC could be identified and deletions could be correctly detected in such samples. However, we are aware that a gain in a sample with MCC might be missed because of the BAF imbalance already present because of MCC. For that reason, we try to exclude blood-stained samples from the diagnostic array assay. ROHs can be detected, which can help to detect uniparental disomy. Despite the high failure rate (20\%) during the validation of the normal control samples (many samples with low DNA input), we decided to conduct our pilot on 61 index fetuses, as we would have enough DNA in pregnancies tested prospectively. In pregnancies with ultrasound abnormalities, our target for array analysis, $30 \mathrm{ml}$ of AF, is sampled in our centre instead of the standard $20 \mathrm{ml}$, which will yield enough DNA in most cases.

A clinically relevant chromosome abnormality was found in $6.5 \%$ (4 out of 61 ) of our index patients with a normal karyotype, which is concordant to the data in the literature. ${ }^{8,17-19}$

In prenatal settings, genomic regions of clinical importance can be overlooked by targeted arrays designed for postnatal studies. ${ }^{8,20}$ Coppinger et $a l^{21}$ demonstrated that whole-genome analysis does not increase the number of results of unclear significance when compared with targeted screening. We have carefully chosen a platform that allows for prenatal whole-genome screening on uAF cells without increasing the number of calls, allowing a quick analysis. HCS is a whole-genome panel designed to detect cytogenetic abnormalities and therefore, probes for some (highly polymorphic) regions are not included. We found about 9.8\% (6 out of 61) CNVs of unknown significance in the index patients group, whereas Tyreman et $a l^{8}$ found $12 \%$ (13 out of 106 ) of them. They tested a similar group; however, they did not test the parental DNA and the array they used (Affymetrix 6.0) has a different (higher) probe coverage, which may explain the differences. Moreover, we have also chosen not to analyse deletions $<150 \mathrm{~kb}$ and duplication $<200 \mathrm{~kb}$ because the capacity to reliably detect single CNVs $<100 \mathrm{~kb}$, appeared to be limited for $\sim 300 \mathrm{k}$ platforms, ${ }^{22}$ and performing high-throughput higher resolution array analysis in such a short time frame might be not feasible. Our approach is a compromise of a rapid screening with a resolution that is much better than conventional karyotyping.

The arguments for implementing HCS as a primary diagnostic tool for fetuses with ultrasound abnormalities were:

- $\quad 50$ ng DNA input; no need for culturing AF cells (most patients are referred in the second trimester), no extra amplification is necessary before the array assay.

- Higher resolution screening results on $\mathrm{UAF}$ is faster than karyotyping.

- No need to take along a control (such as in case of $105 \mathrm{~K} / 180 \mathrm{~K}$ Agilent).

- Detection of CNV, $\mathrm{ROH}$ and polyploidy, and easier recognition of false-positive calls (because of extra information from the BAF).

- Whole-genome analysis does not increase the number of results of unclear significance when compared with targeted screening. ${ }^{21}$

- Low level of mosaicism is easier to determine because of information from the BAF. ${ }^{23}$

After pre-test counselling, the parents had an opportunity to decide which test results they wish to receive (Table 2). None of the couples disagreed on our policy that abnormalities of uncertain or unknown clinical significance (UVs) would not be revealed to them. We decided not to inform the parents about UVs because these findings may be clinically irrelevant for the ongoing pregnancy and may cause anxiety. Although we did not notify the parents about UVs, it is important to collect these data and detailed clinical follow-up of the patients because in the future an association may be found.

Most of the parents were interested in genetic abnormalities influencing the health and development of their unborn child in infancy and childhood. However, about half of the parents also chose to be informed about genetic abnormalities affecting the health of their future child in adulthood. So far we did not detect a CNV that probably has an adverse health effect in adulthood. In case we find one, the parents will receive extensive genetic counselling on possible consequences and will be offered psychological counselling. In the Netherlands, the fetus is legally considered to be part of its mother and therefore not protected by the right not to know. The disclosure of genetic information involving the health in adult life of an unborn individual could be regarded as a violation of its autonomy. Moreover, this information may have implications on the health of one of the parents and possibly also on his or her family. Naturally, these issues are addressed in the pre-test counselling and it will be included in our new informed consent form. More research needs to be carried out on the motivations of the parents in choosing which test results they wish to receive. In addition we have to investigate whether future parents can truly make informed decisions regarding these complex matters in such a small time frame. ${ }^{24}$

\section{CONFLICT OF INTEREST}

The authors declare no conflict of interest. 


\section{ACKNOWLEDGEMENTS}

We thank B Beverloo and J Saris for using the array facility unit, Y van Bever for referring cases and gynaecologists for referring cases to the clinical geneticists.

1 Shaffer LG, Coppinger J, Alliman S et al: Comparison of microarray-based detection rates for cytogenetic abnormalities in prenatal and neonatal specimens. Prenat Diagn 2008; 28: 789-795.

2 Rickman L, Fiegler H, Carter NP, Bobrow M: Prenatal diagnosis by array-CGH. Eur J Med Genet 2005; 48: 232-240.

3 Rickman L, Fiegler H, Shaw-Smith $\mathrm{C}$ et al: Prenatal detection of unbalanced chromosomal rearrangements by array CGH. J Med Genet 2006; 43: 353-361.

4 Sahoo T, Cheung SW, Ward P et al: Prenatal diagnosis of chromosomal abnormalities using array-based comparative genomic hybridization. Genet Med 2006; 8: 719-727.

5 Friedman JM: High resolution array genomic hybridization in prenatal diagnosis. Prenat Diagn 2009; 29: 20-28.

6 Kleeman L, Bianchi D, Shaffer LG et al: Use of array comparative genomic hybridization for prenatal diagnosis of foetuses with sonographic anomalies and normal metaphase karyotype. Prenat Diagn 2009; 29: 1213-1217.

7 Van den Veyver IB, Patel A, Shaw CA et al: Clinical use of array comparative genomic hybridization (aCGH) for prenatal diagnosis in 300 cases. Prenat Diagn 2009; 29: 29-39.

8 Tyreman M, Abbott KM, Willatt LR et al: High resolution array analysis: diagnosing pregnancies with abnormal ultrasound findings. J Med Genet 2009; 46: 531-541.

9 Faas $\mathrm{BH}$, van der Burgt I, Kooper AJ et al: Identification of clinically significant, submicroscopic chromosome alterations and UPD in foetuses with ultrasound anomalies using genome-wide 250 k SNP array analysis. J Med Genet 2010; 47: 586-594.

10 Vermeesch JR, Fiegler $\mathrm{H}$, de Leeuw N: Guidelines for molecular karyotyping in constitutional genetic diagnosis. Eur J Hum Genet 2007; 15: 1105-1114.

11 Murthy SK, Nygren AO, EI Shakankiry HM et al: Detection of a novel familial deletion of four genes between BP1 and BP2 of the Prader-Willi/Angelman syndrome critical region by oligo-array $\mathrm{CGH}$ in a child with neurological disorder and speech impairment. Cytogenet Genome Res 2007; 116: 135-140.

12 Doornbos M, Sikkema-Raddatz B, Ruijvenkamp CA et al: Nine patients with a microdeletion 15q11.2 between breakpoints 1 and 2 of the Prader-Willi critical region, possibly associated with behavioural disturbances. Eur J Med Genet 2009; 52: $108-115$.

13 de Kovel CG, Trucks H, Helbig I et al: Recurrent microdeletions at $15 q 11.2$ and 16 p13.11 predispose to idiopathic generalized epilepsies. Brain 2010; 133 (part 1): 23-32.

14 Lecointre C, Pichon O, Hamel A et al: Familial acampomelic form of campomelic dysplasia caused by a $960 \mathrm{~kb}$ deletion upstream of SOX9. Am J Med Genet A 2009; 149A: $1183-1189$.

15 Kurahashi H, Nakayama T, Osugi $Y$ et al: Deletion mapping of 22q11 in CATCH22 syndrome: identification of a second critical region. Am J Hum Genet 1996; 58 : 1377-1381.

16 Talseth-Palmer BA, Bowden NA, Hill A, Meldrum C, Scott RJ: Whole genome amplification and its impact on CGH array profiles. BMC Res Notes 2008; 1: 56.

17 Le Caignec C, Boceno M, Saugier-Veber P et al: Detection of genomic imbalances by array based comparative genomic hybridisation in foetuses with multiple malformations. J Med Genet 2005; 42: 121-128.

18 Valduga M, Philippe C, Bach Segura $\mathrm{P}$ et al: A retrospective study by oligonucleotide array-CGH analysis in 50 foetuses with multiple malformations. Prenat Diagn 2010; 30: 333-341.

19 Hillman SC, Pretlove S, Coomarasamy A et al: Additional information from array comparative genomic hybridisation (array CGH) technology over conventional karyotyping in prenatal diagnosis-a systematic review and meta-analysis. Ultrasound Obstet Gynecol 2011; 37: 6-14.

20 Veltman JA, de Vries BB: Diagnostic genome profiling: unbiased whole genome or targeted analysis? J Mol Diagn 2006; 8: 534-539.

21 Coppinger J, Alliman S, Lamb AN, Torchia BS, Bejjani BA, Shaffer LG: Whole-genome microarray analysis in prenatal specimens identifies clinically significant chromosome alterations without increase in results of unclear significance compared to targeted microarray. Prenat Diagn 2009; 29: 1156-1166.

22 Vissers LELM, de Vries BBA, Veltman JA: Genomic microarrays in mental retardation: from copy number variation to gene, from research to diagnosis. J Med Genet 2010; 47: 289-297.

23 Conlin LK, Thiel BD, Bonnemann CG et al: Mechanisms of mosaicism, chimerism and uniparental disomy identified by single nucleotide polymorphism array analysis. Hum Mol Genet 2010; 19: 1263-1275.

24 van de Vathorst S, Verhagen AA, Wildschut HI, Wolf $H$, Zeeman GG, Lind J: Termination of pregnancy after the 20-week ultrasonographic examination: haste and caution. Ned Tijdschr Geneeskd 2008; 152: 2589-2591.

Supplementary Information accompanies the paper on European Journal of Human Genetics website (http://www.nature.com/ejhg) 